\title{
Efficient Solution of Inverse Thermal Problem via Parametric Model Order Reduction
}

\author{
Tamara Bechtold ${ }^{(1)}$, Dennis Hohlfeld ${ }^{(2)}$, Evgenii B. Rudnyi ${ }^{(3)}$ \\ (1)MCRTN COMSON, University of Wuppertal, Gauss Str. 20, 42119 Wuppertal, Germany \\ ${ }^{(2)}$ Holst Centre / IMEC, High Tech Campus 31, 5656 AE Eindhoven, The Netherlands \\ (3)CADFEM GmbH, Marktplatz 2, 85567 Grafing b. München, Germany
}

\begin{abstract}
In this paper we present a novel approach to determine material thermal properties of thin film materials. As a testcase we have chosen silicon nitride employed in a micro-hotplate structure. We use ANSYS to build a threedimensional finite element (FE) model of the test structure, which considers heat conduction and convection effects. It is further parameterized and subjected to parametric model order reduction (pMOR). The parameters of the reduced model are the unknown material thermal properties and the heat transfer coefficient, which are automatically adjusted within the optimization loop, so that the simulation results of the model are in agreement with the transient temperature measurements. The use of parameterized reduced order models within the optimization iterations speeds up the transient solution time by several orders of magnitude, while retaining almost the same precision as the full FE model.
\end{abstract}

\section{Introduction}

The design of new microelectromechanical systems (MEMS) requires knowledge of properties of the used materials. This information is well known for most bulk materials. Monocrystaline silicon is the dominant material for MEMS fabrication and has been extensively studied so that its mechanical, thermal and electrical characteristics are precisely known [1],[2]. Devices which are purely made from silicon, e.g. high frequency resonators or oscillating mirrors, can be exactly modelled due to the well known mechanical properties of the material. However, the fabrication of most MEMS devices involves the deposition of thin films, which are employed to fulfill specific functions, like sensing, actuation, passivation, etc. Unfortunately, the material properties of thin films are only partially known, as they strongly vary with fabrication process. In order to build an accurate model of such a structure, the material properties of thin films have to be determined.

In case of electro-thermal MEMS, one needs to determine the material thermal properties of thin film materials. A conventional way to do this is to design, fabricate and characterize dedicated test structures, which feature simple geometry and employ the thin film material under test as a functional component. Simple test structure might include membranes [3] or beams [4] fabricated from a single material. These test structures can be described by analytical models. If the heat source and temperature distribution are known or measured on such test structures, one can determine the material thermal properties of the employed thin films.

Obviously, this approach requires a trade-off between accuracy and ease of use. Simple analytical models assume that heat flows only in one direction from the source to the sink. However, heat conduction is a distributed phenomenon and thus some fractions of the heat flow are neglected. Therefore, the more precise numerical models are required for higher accuracy. However, the resulting equations can only be solved numerically at high computational effort. Furthermore, it is necessary to adjust the model parameters to match the simulation and characterization results. This is usually performed within a time consuming optimization process, which requires the time integration of the numerical model in each iteration [5].

In this work, we propose to use the advanced mathematical algorithms of parametric model order reduction (pMOR) [6] to speed up the optimization of the numerical model and hence, enable accurate and efficient determination of the material thermal parameters of thin films.

\section{MEMS Case Study - Silicon-Based-Microhotplate}

The fabricated structure resembles a micro-hotplate since it features a membrane for thermal isolation and integrated resistors for heat generation (see Fig. 1). This class of structures is employed in a variety of other microfabricated devices such as gas sensors [7] and infrared sources [8].

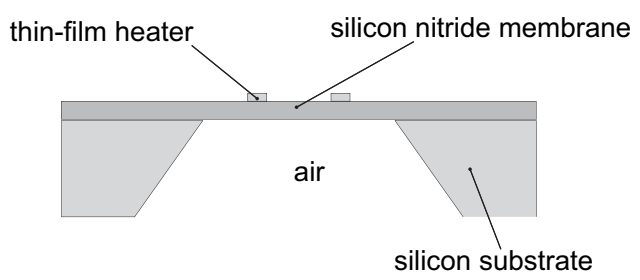

Fig. 1 A silicon-nitride membrane with integrated heater and sensing element was fabricated by low-frequency plasma enhanced chemical vapor deposition. The square membrane is $500 \mathrm{~nm}$ thick with a side length of $550 \mu \mathrm{m}$, and the thin-film heater is made from $150 \mathrm{~nm}$ platinum with a $50 \mathrm{~nm}$ titanium adhesion layer.

The membrane features a thin-film metal resistor for temperature modulation through Joule heating. A second resistor, which serves as a temperature sensor, is placed adjacent to the heater. This resistor is configured for a four-point resistance measurement. In order to achieve a 
preferably circular symmetric and homogenous temperature distribution at the center of the square membrane, both resistors are arranged as shown in Fig. 2.

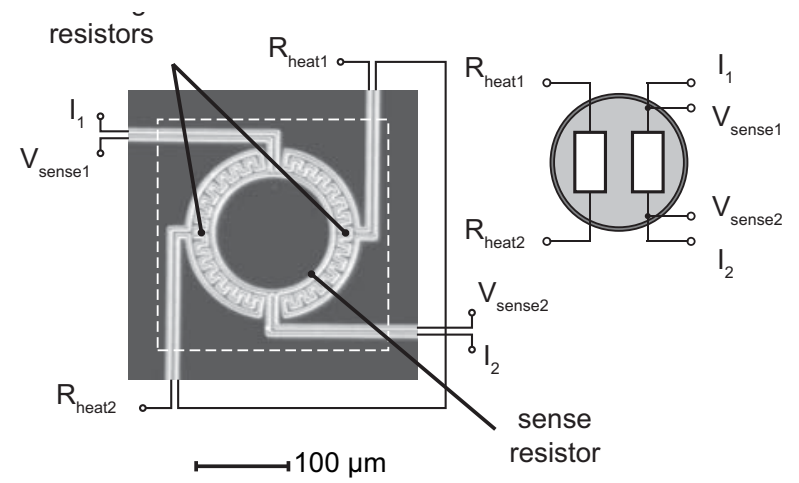

Fig. 2 Schematic view of the thin-film resistors for heating and temperature sensing. Heating resistor is operated at constant voltage. The sensing resistor is configured for four-point measurement.

The characterization of the static and transient thermal properties of the membrane is performed on a temperature controlled mount. For characterization of transient temperature changes, a constant current of $100 \mu \mathrm{A}$ is passed through the sensing resistor while the voltage across the inner terminals is measured using an oscilloscope. In order to use the sensing resistor as a temperature sensor, the linear temperature coefficient of the material's resistivity has to be known. The temperature coefficient is measured by acquiring the sensor's resistance at various temperatures. These are precisely set by the Peltier-mount. The electrical resistance depends linearly on the temperature over the investigated temperature range and is modeled through:

$$
R(\Delta T)=R_{0}(1+\alpha \Delta T)
$$

with $R_{0}$ as the resistance for $\Delta T=0 \mathrm{~K}$. A temperature coefficient of $\alpha=2.29310^{-4} \mathrm{~K}^{-1}$ is obtained for a metallization of $150 \mathrm{~nm}$ platinum with $50 \mathrm{~nm}$ titanium. The transient thermal response of the membrane is characterized by applying a rectangular voltage signal to the heating resistor using a function generator. The signal output is configured as a voltage source with a fixed output impedance of $50 \Omega$. The thermal response over a whole period is presented in Fig. 3. One can recognize the drop in the heating power shortly after the voltage is applied. This is due to the fact that also the heating resistor depends on temperature. An increase in temperature causes the heating resistance to grow which leads to slightly smaller heating power. After applying the heating power, the membrane's temperature increases until a maximum value is reached. This temperature is defined as the steady-state value. After setting the power to zero, the heat stored in the membrane's volume is dissipated to the surrounding media by conduction and free convection. Thus, the temperature drops down to its initial value.

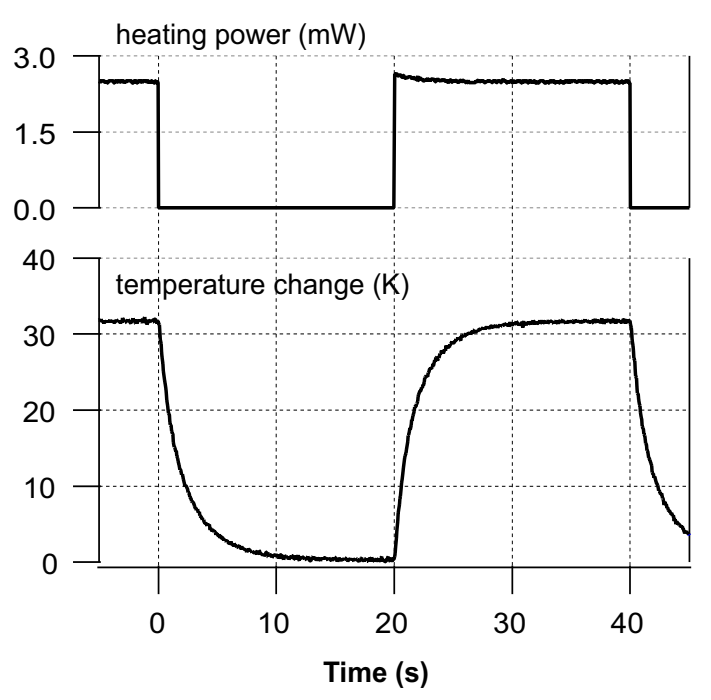

Fig. 3 Temperature modulation of a silicon/nitride membrane. A square wave heating power with a frequency of $25 \mathrm{~Hz}$ was applied to the membrane.

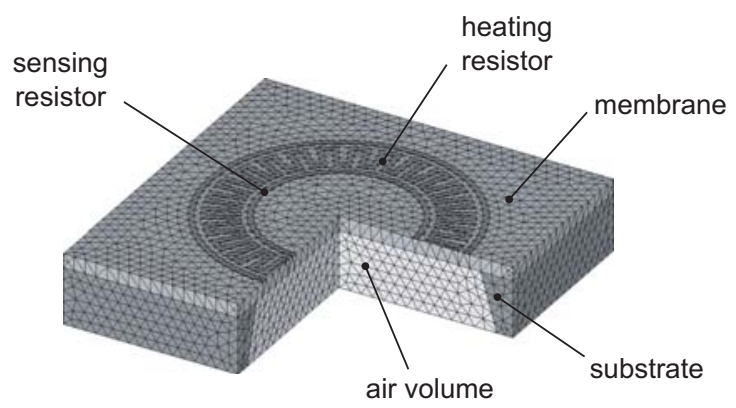

Fig. 4 FE mesh of the three-dimensional model with 66.000 nodes.

We use a full 3D model with 66.000 nodes (see Fig. 4), which considers the heat conduction through the solid material and the air beneath the membrane as well as convection to the air above the membrane. Fig. 5 shows the considered heat loss mechanisms in more detail.

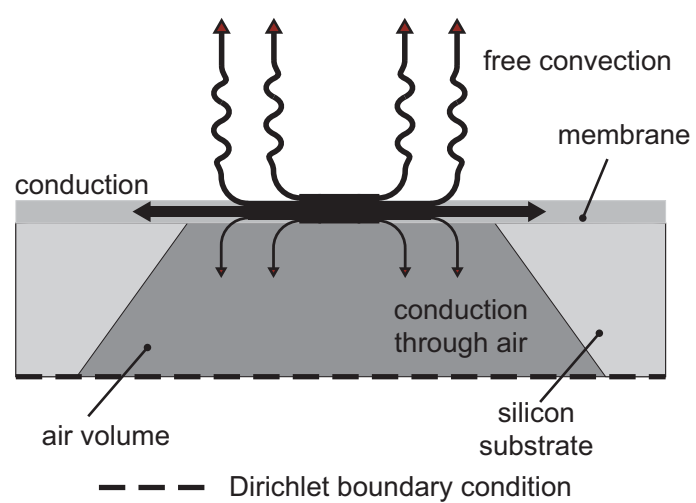

Fig. 5 Heat loss mechanisms considered by the FE model. Dirichlet boundary condition $T=0^{\circ} \mathrm{K}$ is set at the bottom edge of the simulation domain. 


\section{Parametric Model Order Reduction}

Mathematical methods of model order reduction (MOR) enable a formal transformation of the physical model, that is, governing partial differential equation to a low-dimensional ordinary differential equation (ODE) system (Fig. 6).
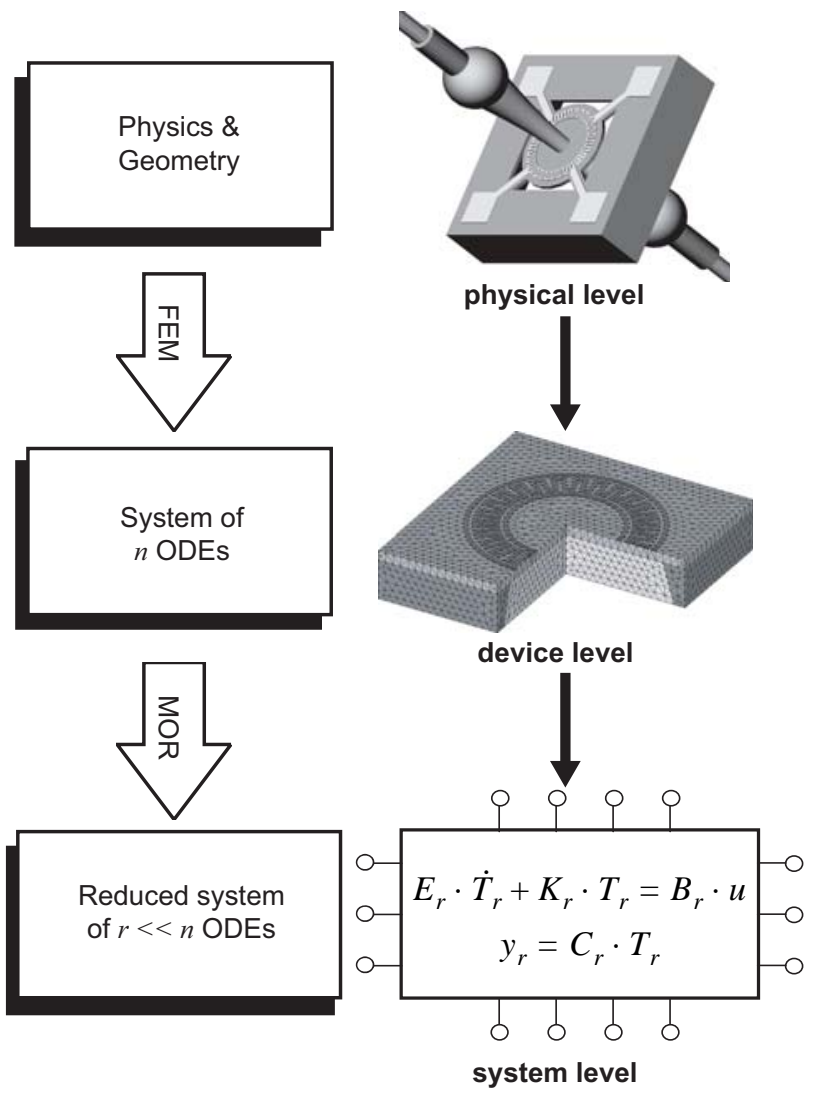

Fig. 6 Place of model order reduction within a conversion process from physical to compact model.

The intermediate level is a device level, that is, a high dimensional ODE system. The first conversion of the physical to the device model we perform via the finite element discretisation. The heat transfer within a hotplate is described through the following equations:

$$
\nabla \bullet(\kappa \nabla T)+Q-\rho c_{p} \frac{\partial T}{\partial t}=0, Q=j^{2} R
$$

where $\kappa(r)$ is the thermal conductivity in $\mathrm{W} / \mathrm{m} / \mathrm{K}$ at the position $r, c_{p}(r)$ is the specific heat capacity in $\mathrm{J} / \mathrm{kg} / \mathrm{K}$, $\rho(r)$ is the mass density in $\mathrm{kg} / \mathrm{m}^{3}, T(r, t)$ is the temperature distribution and $Q(r, t)$ is the heat generation rate per unit volume in $\mathrm{W} / \mathrm{m}^{3}$. We solve (2) with the initial condition $T_{0}=0$, the Dirichlet boundary condition $T=0$ at the bottom of computational domain and the convection boundary condition at the top of the membrane:

$$
q=h\left(T-T_{\text {air }}\right)
$$

where $h$ is the heat transfer coefficient between the membrane and the ambient air in $\mathrm{W} / \mathrm{m}^{2} / \mathrm{K}$. Assuming that the heat generation is uniformly distributed within the heater and that the system matrices can be considered as temperature independent at the operation temperature, the finite element based spatial discretization of (2) leads to a large linear ODE system of the form:

$$
\begin{gathered}
E \cdot \dot{T}+K \cdot T=B \cdot \frac{U^{2}(t)}{R(T)} \\
y=C \cdot T
\end{gathered}
$$

where $E$ and $K$ are the global heat capacity and heat conductivity matrices, $B$ is the load vector (matrix) and $C$ is the output vector (matrix). Equation (4) is a starting point for model order reduction, which leads to a system of the same form but with much smaller dimension. In the engineering problems, the most often used MOR approach is a moment matching approach [9], which is based on approximation of the transfer function

$$
H(s)=C(s E+K)^{-1} B
$$

as (4) a Taylor series around some value of the Laplace variable $s=s_{0}$ :

$$
H(s)=\sum_{i=0}^{\infty} m_{i}\left(s_{0}\right)\left(s-s_{0}\right)^{i}
$$

where $m_{i}\left(s_{0}\right)=C\left(-\left(K+s_{0} E\right)^{-1} E\right)^{i} \cdot\left(K+s_{0} E\right)^{-1} B \quad$ is called the $i$-th moment around $s_{0}$, and then finding a much lower order $r$ system whose transfer function $H_{r}(s)$ has the same moments as $H(s)$ up to the degree $r$.

Note that in its original form model reduction does not allow to preserve parameters within the system matrices, which arise naturally in many applications, in the form of e.g. boundary conditions, material parameters or geometry parameters of the device. For microhotplate (4) under the convection boundary condition (3) and assuming $T_{\text {air }}$ to be zero, can be rewritten as:

$$
\begin{gathered}
\left(E_{0}+\rho c_{p} \cdot E_{1}\right) \dot{T}+\left(K_{0}+\kappa \cdot K_{1}+h \cdot K_{2}\right) T=b \frac{U^{2}(t)}{R(T)} \\
y=C^{T} \cdot T
\end{gathered}
$$

Here, it is important to emphasize that all system matrices depend linearly on the three parameters, volumetric heat capacity $c_{p} \cdot \rho$, thermal conductivity $\kappa$ and the heat transfer coefficient $h$ between the membrane and the ambient air. Therefore they can be factorized. From a numerical point of view, every parameter $p_{i}$ in the transfer function:

$$
H\left(s, p_{i}\right)=C\left[s\left(E_{0}+\rho c_{p} E_{1}\right)+K_{0}+\kappa K_{1}+h K_{2}\right]^{-1} B
$$


of (7) is "equivalent" to the Laplace variable s. Hence, the first idea explored by several groups [10] - [13] and based on generalization of moment matching, was to make multivariate expansions of (8) with respect to the Laplace variable $s$ and parameters $p_{i}$ which are thus simultaneously preserved in symbolic form.

We have used the multivariate-moment-matching approach from [13] and were able to reduce the time for transient integration of the FE model by a factor of 100 . A parametric reduced model has only 117 degrees of freedom (DOF) but still provides high accuracy (see Fig. 7).
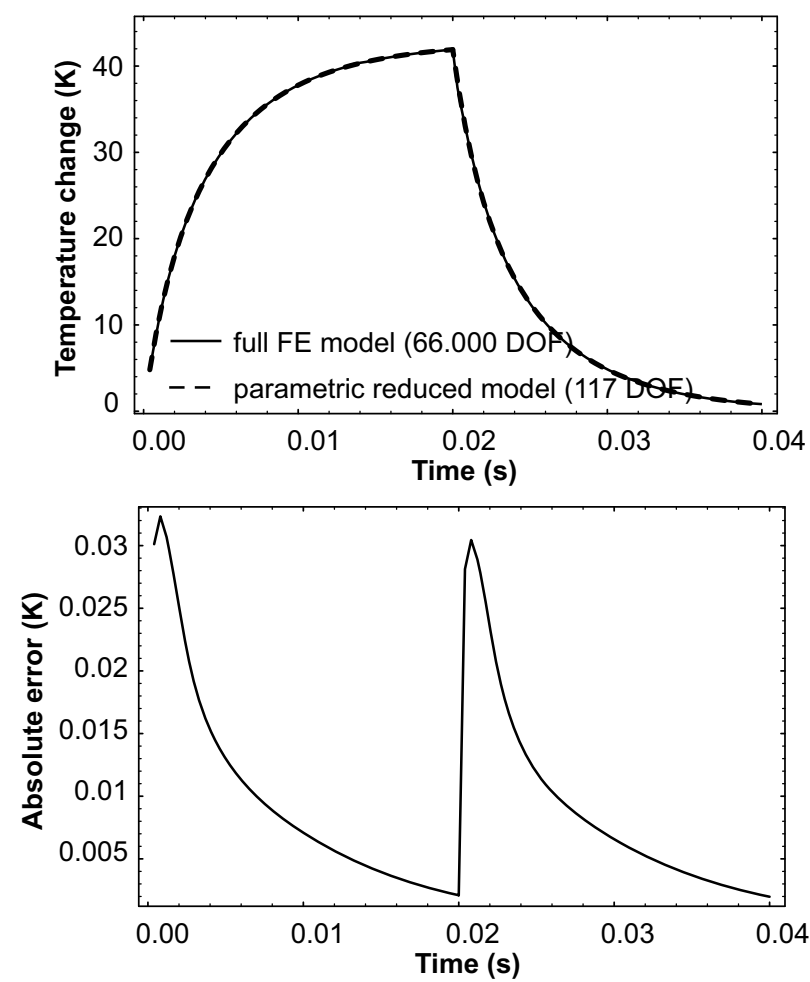

Fig. 7 Comparison between the full FE model with 66. 000 DOF and the parametric reduced model with 117 DOF for the initial parameter values.

\section{Optimization Results}

Because material parameters and the heat transfer coefficient are preserved as parameters within the reduced model, they can be altered in each iteration of the optimization process. By defining an objective function, which characterizes the difference between simulated and measured results, data fitting cycle according to the algorithm in Fig. 8 is performed. Note that, thanks to pMOR, no new FE model must be built and reduced in each iteration, as done in [17] and [18].

Fig. 9 shows the comparison between the measured temperature curve and the initial FE model, in which the thermal properties have been set to literature values from Table 1 . The temperature dependence of the material parameters has been neglected, as the measurement results were obtained at comparatively low temperatures.

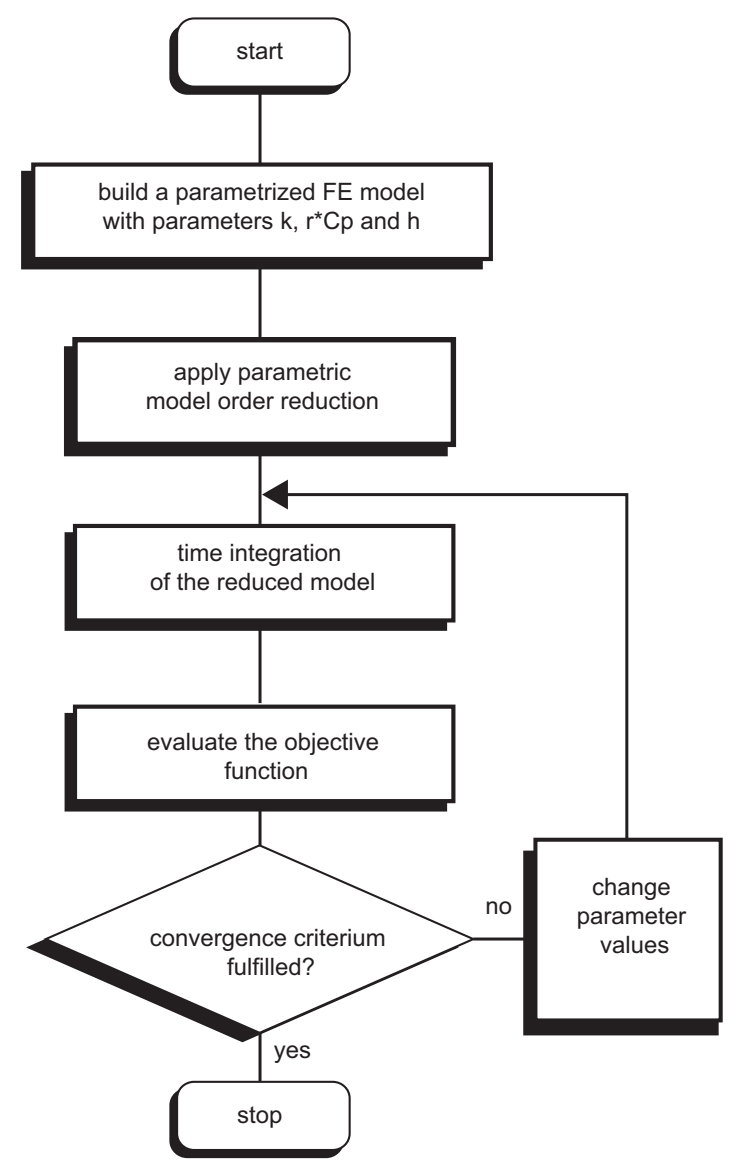

Fig. 8 Algorithm for fast determination of material properties via parametric model order reduction. Parameterized FE model is created by extracting the system matrices from ANSYS via software tool MOR for ANSYS [14]. pMOR is done in mathematica [15] and optimization with DOT optimizer [16].

Table 1 Material parameters

\begin{tabular}{|r|c|c|c|}
\hline & $\begin{array}{c}\text { initial guess } \\
\text { from [19] }\end{array}$ & $\begin{array}{c}\text { optimization } \\
\text { result }\end{array}$ \\
\hline $\begin{array}{r}\text { heat } \\
\text { conductivity }\end{array}$ & $\kappa / \frac{W}{\mathrm{~m} \cdot K}$ & 2.5 & 4.2 \\
\hline $\begin{array}{r}\text { specific heat } \\
\text { capacity }\end{array}$ & $c_{p} / \frac{\mathrm{J}}{\mathrm{kgK}}$ & 750 & 439 \\
\hline density & $\rho / \frac{\mathrm{kg}}{\mathrm{m}^{3}}$ & 3100 & 3100 \\
\hline film coefficient & $h / \frac{\mathrm{W}}{\mathrm{m}^{2} K}$ & 10 & 11.4 \\
\hline
\end{tabular}

Fig. 10 shows the measured and the simulated temperature response after 40 cycles of optimization. An optimum fit could be obtained for the parameter values from Table 1. These parameters result in an excellent match 
between the model and the measurements. Fig. 11 shows further the variation of each parameter during the course of optimization.

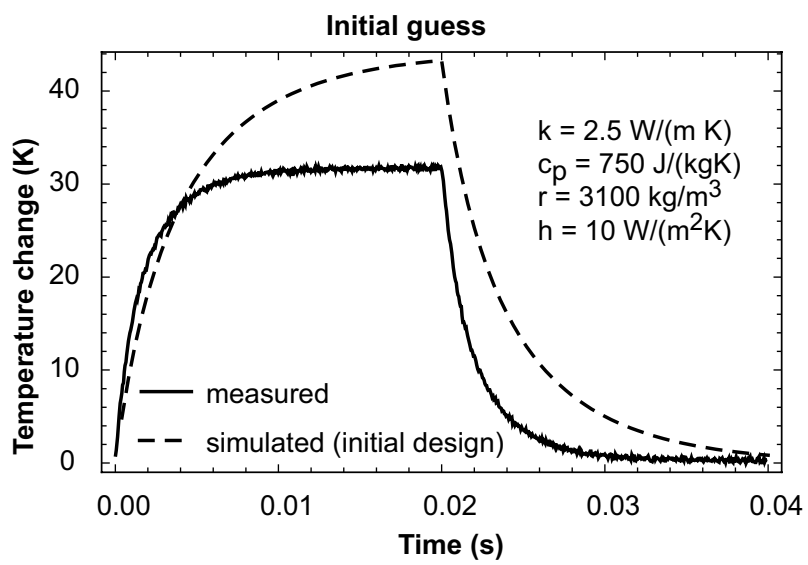

Fig. 9 Measured and simulated transient curves for the initial parameter values.

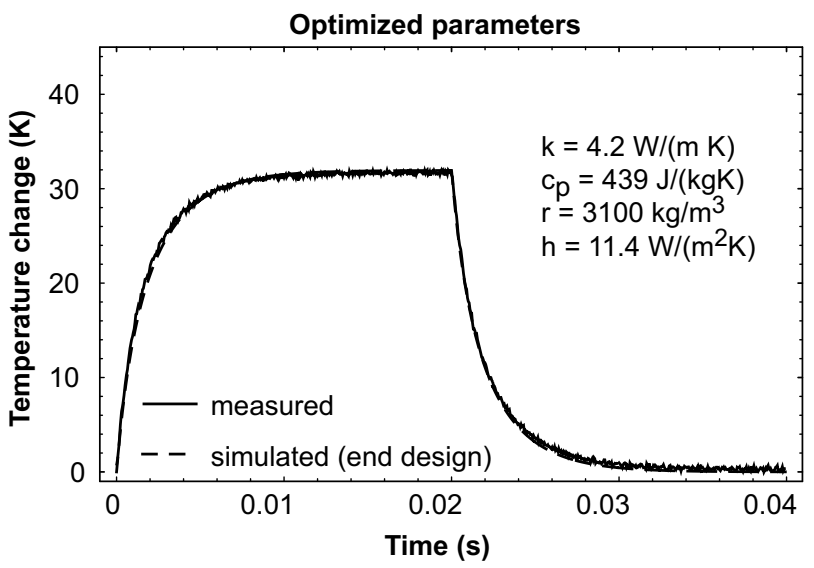

Fig. 10 Measured and simulated transient curves after 55 cycles of optimization.

\section{Conclusions and Outlook}

We have demonstrated a methodology for the solution of the inverse thermal problem via parametric model order reduction of finite element models and subsequent automatic parameter optimization. We were able to extract material thermal parameters as heat conductivity, heat capacity and convection film coefficient from transient thermal characterization results in the most efficient way, while using highly accurate three-dimensional numerical model of the test structure. The data was obtained from a silicon-nitride microhotplate.

The next step will be to apply this methodology to the material properties identification for electrothermal structures which are composed of several different materials, like e.g. composite membranes.
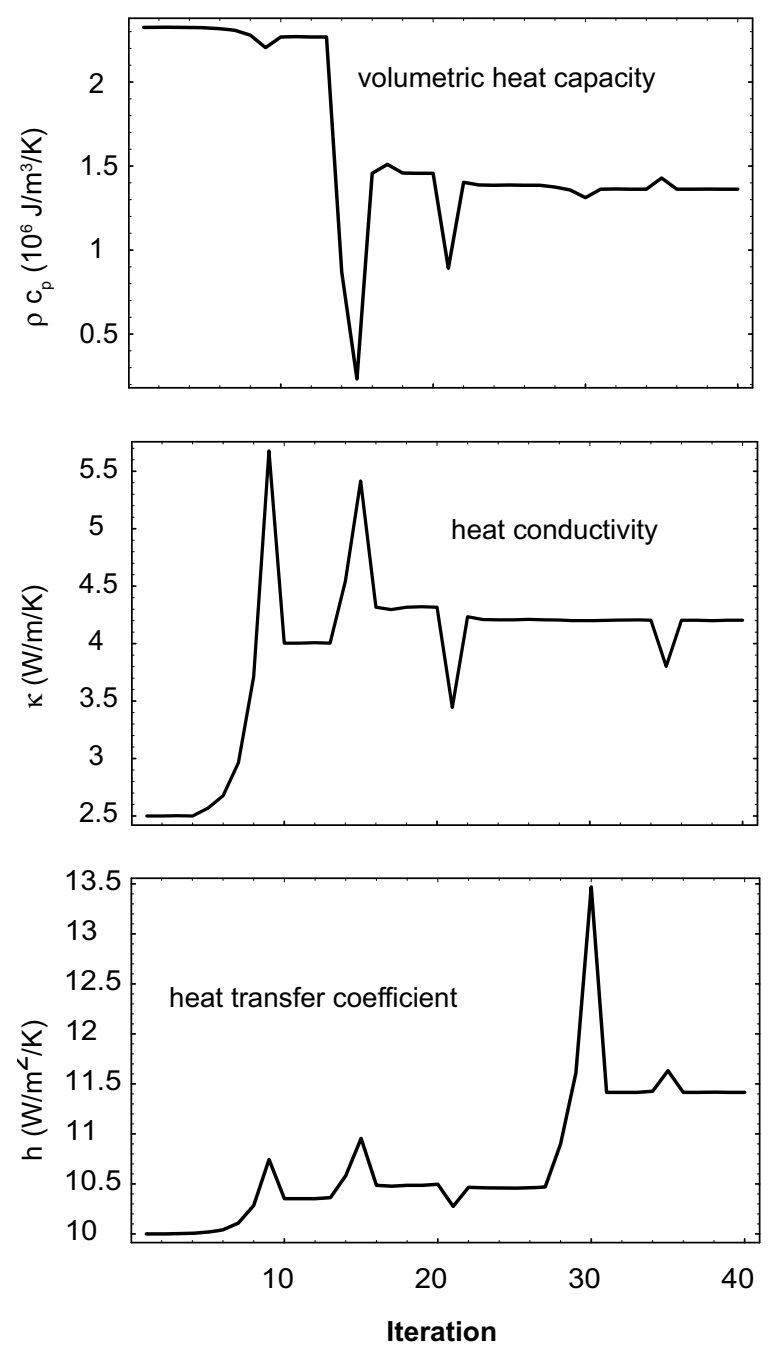

Fig. 11 Parameter development during the course of optimization.

\section{References}

1. K. E. Petersen, "Silicon as a mechanical material," Proceedings of the IEEE, Vol. 70, pp. 420-457, 1982.

2. R. Hull (ed.), Properties of Crystalline Silicon, Institution of Engineering and Technology, 1999.

3. F. Volklein and T. Starz, "Thermal conductivity of thin films-experimental methods and theoretical interpretation," Proceedings XVI International Conference on Thermoelectrics, 1997, pp. 711-718.

4. A. Roncaglia, E. Cozzani, F. Mancarella, M. Passini, G. C. Cardinali, and M. Severi, "Influence of Air Heat Exchange Upon On-Chip Measurement of Thermal Conductivity using MEMS Test Structures," Proc. of International Solid-State Sensors, Actuators and Microsystems Conference (TRANSDUCERS), 2007, pp. 615-618. 
5. N. Stojanovic, Y. Jongsin, E. B. K. Washington, J. M. Berg, M. W. Holtz, and H. Temkin, "Thin-Film Thermal Conductivity Measurement Using Microelectrothermal Test Structures and Finite-Element-ModelBased Data Analysis," Microelectromechanical Systems, Journal of, vol. 16, pp. 1269-1275, 2007.

6. L. H. Feng, "Parameter independent model order reduction", Mathematics and Computers in Simulation, vol. 68, no. 3, pp. 221-234, 2005.

7. M. Graf, D. Barrettino, S. Taschini, C. Hagleitner, A. Hierlemann, and H. Baltes, "Metal Oxide-Based Monolithic Complementary Metal Oxide Semiconductor Gas Sensor Microsystem," Analytical Chemistry, vol. 76, pp. 4437-4445, 2004.

8. J. Spannhake, O. Schulz, A. Helwig, G. Miiller, and T. Doll, "Design, development and operational concept of an advanced MEMS IR source for miniaturized gas sensor systems," in Sensors, 2005 IEEE, 2005.

9. R. W. Freund, "Krylov-subspace methods for reducedorder modeling in circuit simulation", Journal of Computational and Applied Mathematics, vol. 123, pp. 395-421, 2000.

10. L. Codecasa, D. D'Amore, and P. Maffezzoni, "A novel approach for generating boundary condition independent compact dynamic thermal networks of packages", Proc. 10th International Workshop on Thermal Investigations of ICs and Systems (THERMINIC), 2004, pp. 305-310.

11. E. B. Rudnyi, L. H. Feng, M. Salleras, S. Marco, J. G. Korvink, "Error Indicator to Automatically Generate Dynamic Compact Parametric Thermal Models", Proc. 11th International Workshop on Thermal Investigations of ICs and Systems (THERMINIC), 2005, pp. 139-145.
12. L. H. Feng, E. B. Rudnyi, J. G. Korvink, "Preserving the film coefficient as a parameter in the compact thermal model for fast electro-thermal simulation", IEEE Transactions on Computer-Aided Design of Integrated Circuits and Systems, vol. 24, no. 12, pp. 1838-1847, 2005.

13. D. Celo, P. K. Gunupudi, R. Khazaka, D. J. Walkey, T. Smy, M. S. Nakhla, "Fast Simulation of Steady-State Temperature Distributions in Electronic Components Using Multidimensional Model Reduction", IEEE Transactions on Components and Packaging Technologies, vol. 28, no. 1, pp. 70-79, 2005.

14. E. B. Rudnyi, J. G. Korvink, "Model Order Reduction for Large Scale Engineering Models Developed in ANSYS," Lecture Notes in Computer Science, vol. 3732, pp. 349-356, 2006.

15. http://modelreduction.com/ModelReduction/Parametric.html

16. DOT Users Manual version 4.20 (Colorado Springs, CO: Vanderplaats Research and Development), at http://www.vrand.com/DOT.html

17. T. Bechtold, D. Hohlfeld, E. B. Rudnyi, H. Zappe, J. G. Korvink, "Inverse Thermal Problem via Model Order Reduction: Determining Material Properties of a Microhotplate”, Proc. 11th International Workshop on Thermal Investigations of ICs and Systems (THERMINIC), 2005, pp. 146-150.

18. J. S. Han, E. B. Rudnyi, J. G. Korvink, "Efficient optimization of transient dynamic problems in MEMS devices using model order reduction”, J. Micromech. Microeng., Vol. 15, No. 4, pp. 822-832, 2005.

19. J. F. Shackelford and W. Alexander, CRC Materials Science and Engineering Handbook: CRC Press, 2000. 